\title{
Response of a Permanent Magnet Moving Coil Instrument via the Application of Rohit Transform
}

\author{
Rohit Gupta $^{* 1}$, Rahul Gupta ${ }^{2}$, Anamika ${ }^{3}$ \\ ${ }^{1,2}$ Lecturer of Physics, Department of Applied Sciences, Yogananda College of Engineering and Technology, Jammu, J\&K, India \\ ${ }^{3}$ Assistant Professor, Department of Electrical Engineering, Yogananda College of Engineering and Technology, Jammu, J\&K, India
}

\begin{abstract}
In this paper, a new integral transform called Rohit transform is presented for the analysis of a Permanent Magnet Moving Coil (PMMC) instrument. A permanent magnet moving coil instrument is an electromagnetic device that is used to measure small values of electric currents. When some current is passed through the permanent-magnet moving-coil (PMMC) instrument, its coil may suffer a few back and forth oscillations about its final mean position before coming to rest. The PM MC instrument is also known as moving coil galvanometer and its mathematical analy sis is usually done by an ordinary calculus approach. This paper presents the use of Rohit transform for mathematical analysis of a PMMC instrument and hence, for obtaining its response. The response obtained provides the deflection of the coil of the PMMC instrument from its mean position. In this paper, the response of a PMMC instrument is provided as a demonstration of the application of the new integral transform called Rohit transform.
\end{abstract}

Keywords - Response; Rohit Transform; Permanent Magnet Moving Coil Instrument.

\section{Introduction}

A permanent-magnet moving-coil (PMMC) instrument also known as moving-coil galvanometer is an electromagnetic device that is used to measure small values of electric currents. It consists of a coil wrapped over a nonmetallic framewhich has a soft iron core, permanent horseshoe magnets, pivoted spring, scale, and pointer. When some current is passed through thepermanent-magnet moving-coil (PMMC) instrument, its coil may suffer a few back and forth oscillations about its final mean position before coming to rest. As the coil suffers deflection, it moves in a permanent magnetic field and therefore, an e.m.f. is induced in it, which opposes the motion of the coil. The electro magnetic damping, responsible for the damping of coil, can be increased by winding the coil on a metallic frame. When the coil rotates, eddy currents are produced in the frame moving along with the coil, which tends to damp its motion and hence the coil soon comes to rest [1-5]. The Rohit transform is a new integral transform which has been recently put forward by the author Rohit Gupta. Ithas been applied in science and engineering to solvemost of the initial value problems [6]. The analysis of a permanent-magnet moving-coil (PMMC) instrument also known as moving-coil galvanometer is usually done by ordinary calculus approach [1-5]. This paper proves the applicability of Rohit Transform for obtaining the response of the PMMC instrument and concludes that Rohit transform like other methods or approaches is an effective and simple tool for obtaining the response of the PMMC instrument also known as moving-coil galvanometer.
The Rohit Transform [6-11] (RT) of $\mathrm{g}(\mathrm{y})$, denoted byR $\{\mathrm{g}(\mathrm{y})\}$, is defined as $\mathrm{R}\{\mathrm{g}(\mathrm{y})\}=r^{3} \int_{0}^{\infty} e^{-r y} g(y) d y$, provided that the integral is convergent, where $r$ may be a real or complex parameter. The Rohit Transform of some of the derivatives [7-10] of a function is

$R\left\{g^{\prime}(y)\right\}=r R\{g(y)\}-r^{3} g(0)$, $R\left\{g^{\prime \prime}(y)\right\}=r^{2} R\{g(y)\}-r^{4} g(0)-r^{3} g^{\prime}(0)$ and so on.

\section{Material And Method}

When some current is passed through the coil of the PMMC instrument, it is turned by the deflecting couple acting on it, and if $\Theta$ is the deflection of the coil from the equilibrium position at any instant $t$, then the motion of thecoil is opposed by the following couples [1-5]:

i) Damping couple $\left(T_{d}\right)$ i.e. $T_{d}=-r \dot{\theta}(t)$, where $r$ is damping constant and the negative sign indicates that the motion of the coil is opposed by the damping couple.

ii) $\left(\mathrm{T}_{\mathrm{d}}\right)$ i.e. $\mathrm{T}_{\mathrm{d}}=-\mathrm{r} \dot{\theta}(\mathrm{t})$,

iii) Restoring couple $\left(\mathrm{T}_{\mathrm{r}}\right)$ i.e. $\mathrm{T}_{\mathrm{s}}=-\mathrm{C} \theta(\mathrm{t})$, where $C$ is torsional rigidity of suspension fibre, and the minus sign shows that the motion is also opposed by the restoring couple.

iv) A couple $\tau_{\mathrm{e}}=-\frac{\mathrm{K}}{R} \dot{\theta}(\mathrm{t})$ arises due to electromagnetic damping i.e. due to induced eddy currents in the coil and depends directly upon the angular velocity of the coil and inversely upon its resistance $\mathrm{R}$ and also depends upon the magnetic field strength. All these factors are included in the constant $\mathrm{K}$.

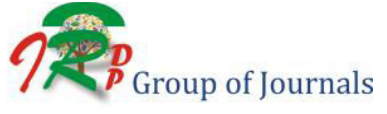


The application of Newton's second law of motion provides the equation of motion of the coil as follows $I \ddot{\theta}(t)=-r \dot{\theta}(t)-C \theta(t)-\frac{K}{R} \dot{\theta}(t)$, where $I$ is the moment of inertia of the coil about its axis of rotation and $\ddot{\theta}(t)$ is its angular acceleration.

The above equation can be rewritten as

$\ddot{\theta}(t)=-\frac{F}{I} \dot{\theta}(t)-\frac{C}{I} \theta(t)-\frac{K}{I R} \dot{\theta}(t)$

Or

$\ddot{\theta}(\mathrm{t})+\left(\frac{\mathrm{r}}{\mathrm{I}}+\frac{\mathrm{K}}{\mathrm{IR}}\right) \dot{\theta}(\mathrm{t})+\frac{\mathrm{C}}{\mathrm{I}} \theta(\mathrm{t})=0 \ldots .(7)$

For convenience let us put $\left(\frac{\mathrm{F}}{\mathrm{I}}+\frac{\mathrm{K}}{\mathrm{IR}}\right)=2 \delta$ and $\frac{\mathrm{C}}{\mathrm{I}}=\omega^{2}$, then equation (7) can be rewritten as

$\ddot{\theta}(\mathrm{t})+2 \delta \dot{\theta}(\mathrm{t})+\omega^{2} \theta(\mathrm{t})=0 \ldots(8)$

The equation (8) is known as a differential equation of the PMMC instrument.

To solve equation (8), the initial boundary conditions are as follows [1-5]:

(i) If the maximum deflection of the coil from the equilibrium position is assumed to be $\theta_{o}$ and we measure the time from the instant when the coil is at the position of its maximu $m$ deflection, then at $\mathrm{t}=0, \theta(0)=\theta_{\mathrm{o}}$.

(ii) At the instant $t=0$, the angular velocity $\dot{\theta}(0)=0$ as the coil is at rest at the instant $t=0$.

The Rohit transform [8, 9] of equation (8) provides $r^{2} \bar{\theta}(\mathrm{r})-r^{4} \theta(0)-r^{3} \dot{\theta}(0)+2 \delta\left\{r \bar{\theta}(\mathrm{r})-r^{3} \theta(0)\right\}+$ $\omega^{2} \bar{\theta}(\mathrm{r})=0 \ldots \ldots .$.

Here $\bar{\theta}(r)$ denotes the Rohit transform of $\theta(t)$.

Applying initial conditions [5] $\theta(0)=\theta_{0}$ and $\dot{\theta}(0)=0$, equation (9) becomes,

$$
\begin{aligned}
& \quad r^{2} \bar{\theta}(r)-r^{4} \theta_{o}+2 \delta\left\{r \bar{\theta}(r)-r^{3} \theta_{o}\right\}+\omega^{2} \bar{\theta}(r)=0 \\
& \text { Or } \\
& {\left[r^{2}+2 \delta r+\omega^{2}\right] \bar{\theta}(r)=\left[r^{4}+2 \delta r^{3}\right] \theta_{o}} \\
& \text { Or } \\
& \bar{\theta}(q)=\frac{\left[r^{4}+2 \delta r^{3}\right] \theta_{o}}{r^{2}+2 \delta r+\omega^{2}}
\end{aligned}
$$$$
\text { Or }
$$$$
\text { Or }
$$

Or

$$
\bar{\theta}(\mathrm{q})=\frac{\left[r^{4}+2 \delta r^{3}\right] \theta_{\mathrm{o}}}{(r+\delta)^{2}-\sqrt{\delta^{2}-\omega^{2}}}
$$

Or

$\bar{\Theta}(\mathrm{q})=\frac{\left[r^{4}+2 \delta r^{3}\right] \theta_{\mathrm{o}}}{\left(r+\delta+\sqrt{\delta^{2}-\omega^{2}}\right)\left(r+\delta-\sqrt{\delta^{2}-\omega^{2}}\right)} \ldots \ldots$.

For convenience let us substitute $\delta+\sqrt{\delta^{2}-\omega^{2}}=$ $\beta_{1}$ and $\delta-\sqrt{\delta^{2}-\omega^{2}}=\beta_{2}$ such that $\beta_{1}-\beta_{2}=2 \sqrt{\delta^{2}-\omega^{2}}$, then equation (10) can be re written as, $\bar{\theta}(r)=\frac{\left[r^{4}+2 \delta r^{3}\right] \theta_{o}}{\left(r+\beta_{1}\right)\left(r+\beta_{2}\right)}$

Or

$\bar{\theta}(\mathrm{r})=\frac{\left[-\beta_{1}+2 \delta\right] r^{3} \theta_{\mathrm{o}}}{\left(-\beta_{1}+\beta_{2}\right)\left(r+\beta_{1}\right)}+\frac{\left[-\beta_{2}+2 \delta\right] r^{3} \theta_{\mathrm{o}}}{\left(-\beta_{2}+\beta_{1}\right)\left(r+\beta_{2}\right)}$

Or

$\bar{\theta}(r)=\frac{\left[-\delta-\sqrt{\delta^{2}-\omega^{2}}+2 \delta\right] r^{3} \theta_{0}}{-2 \sqrt{\delta^{2}-\omega^{2}}\left(r+\beta_{1}\right)}+\frac{\left[-\delta+\sqrt{\delta^{2}-\omega^{2}}+2 \delta\right] r^{3} \theta_{0}}{2 \sqrt{\delta^{2}-\omega^{2}}\left(r+\beta_{2}\right)}$

Or

$\bar{\theta}(r)=-\frac{\left[\delta-\sqrt{\delta^{2}-\omega^{2}}\right] r^{3} \theta_{\mathrm{o}}}{2 \sqrt{\delta^{2}-\omega^{2}}\left(r+\beta_{1}\right)}+\frac{\left[\delta+\sqrt{\delta^{2}-\omega^{2}}\right] r^{3} \theta_{\mathrm{o}}}{2 \sqrt{\delta^{2}-\omega^{2}}\left(r+\beta_{2}\right)} \ldots \ldots$

The application of inverse Rohit transform $[6,12,13$, provides

$\theta(t)=-\frac{\left[\delta-\sqrt{\delta^{2}-\omega^{2}}\right] \theta_{\mathrm{o}} e^{-\beta_{1} t}}{2 \sqrt{\delta^{2}-\omega^{2}}}+\frac{\left[\delta+\sqrt{\delta^{2}-\omega^{2}}\right] \Theta_{\mathrm{o}} e^{-\beta_{2} t}}{2 \sqrt{\delta^{2}-\omega^{2}}}$

Or

$\theta(\mathrm{t})=$

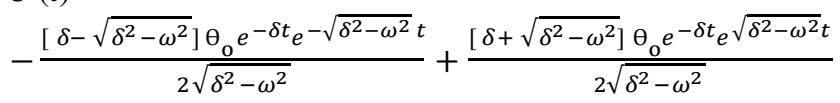

Or

$\theta(\mathrm{t})=$

$\frac{\Theta_{0} e^{-\delta t}}{2}\left\{\left(1+\frac{\delta}{\sqrt{\delta^{2}-\omega^{2}}}\right) e^{\sqrt{\delta^{2}-\omega^{2}} t}+(1-\right.$

$\left.\frac{\delta}{\sqrt{\delta^{2}-\omega^{2}}}\right) e^{-\sqrt{\delta^{2}-\omega^{2}} t}$

This equation (12) provides the deflection of the coil of the PMMC instrument and reveals that the nature of its deflection depends on the nature of the quantity $\sqrt{\delta^{2}-\omega^{2}}$ which may be real, zero or imag inary depending upon the values of $\delta$ and $\omega$. We have the following three cases:

Case I: When $\delta>\omega$, thenthe quantity $\sqrt{\delta^{2}-\omega^{2}}$ is real and therefore, the equation (12) can be rewritten as

$$
\begin{aligned}
& \Theta(\mathrm{t})=\Theta_{\mathrm{o}} e^{-\delta t}\left[\frac{\delta}{\sqrt{\delta^{2}-\omega^{2}}} \sinh \sqrt{\delta^{2}-\omega^{2}} t+\right. \\
& \left.\cosh \sqrt{\delta^{2}-\omega^{2}} t\right] \ldots .(13)
\end{aligned}
$$

It is clear from the equation (13) that the motion of the coil of the PMMC instrument is non-oscillatory and the coil approaches equilibrium quite slowly without any oscillation when a steady current is passed through it.The galvanometer in such a case is said to be over-damped or dead beat $[5,15]$.

Case II: When $\delta=\omega$,then the quantity $\sqrt{\delta^{2}-\omega^{2}}$ is zero. In this case, equation (12) reveals that the motion of the coil ofthe PMMC instrumentis indeterminate, which is not possible. If the quantity $\sqrt{\delta^{2}-\omega^{2}}$ is so small that it approaches zero, then on expanding the exponential terms

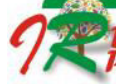


containing the quantity $\sqrt{\delta^{2}-\omega^{2}}$ and neglecting higher order terms, we can rewrite equation (12) as

$\Theta(\mathrm{t})=\theta_{\mathrm{o}} e^{-\delta t}\left\{\frac{\delta}{\sqrt{\delta^{2}-\omega^{2}}} \frac{1+\left(\sqrt{\delta^{2}-\omega^{2}}\right) \mathrm{t}-\left[1-\left(\sqrt{\delta^{2}-\omega^{2}}\right) \mathrm{t}\right]}{2}+\right.$
$\left.\frac{1+\left(\sqrt{\delta^{2}-\omega^{2}}\right) \mathrm{t}+\left[1-\left(\sqrt{\delta^{2}-\omega^{2}}\right) \mathrm{t}\right]}{2}\right\}$

Or $\theta(t)=\theta_{0}(1+\delta t) e^{-\delta t}$

It is clear from the equation (14) that the motion of the coil of the PMMC instrument is non - oscillatory and the coil approaches equilibrium as fast as possible without any oscillation when a steady current is passed through it. The galvanometer in such a case is said to be critically damped [5, 16]. This type of damping is very desirable feature in the PMMC instrument.

Case III: In the case of light damping [5],$\delta<\omega$. In such a case, the quantity $\sqrt{\delta^{2}-\omega^{2}}$ is imaginary. We can rewrite the quantity $\sqrt{\delta^{2}-\omega^{2}}$ as $\sqrt{\delta^{2}-\omega^{2}}=\mathrm{i} \sqrt{\omega^{2}-\delta^{2}}$ (15)

Using equation (15), we can rewrite equation (12) as

$\theta(\mathrm{t})=$

$\Theta_{\mathrm{o}} e^{-\delta t}\left[\frac{\delta}{\sqrt{\omega^{2}-\delta^{2}}} \sin \sqrt{\omega^{2}-\delta^{2}} t+\right.$

$\left.\cos \sqrt{\omega^{2}-\delta^{2}} t\right] \ldots \ldots \ldots \ldots(16)$

Let us substitute $\frac{\theta_{\mathrm{o}} \delta}{\sqrt{\omega^{2}-\delta^{2}}}=\mathcal{A} \cos \varphi$ and $\Theta_{\mathrm{o}}=\mathcal{A} \sin \varphi$ such that $\mathcal{A}=\frac{\theta_{0} \omega}{\sqrt{\omega^{2}-\delta^{2}}}$ and $\varphi=\tan ^{-1} \frac{\sqrt{\omega^{2}-\delta^{2}}}{\delta}$, then equation (16) becomes

$\theta(\mathrm{t})=\mathcal{A} e^{-\delta t} \sin \left[\left(\sqrt{\omega^{2}-\delta^{2}}\right) \mathrm{t}+\varphi\right]$

It is clear from the equation (17) that the motion of the coil of the PMMC instrument is oscillatory with amplitude $\mathcal{A} e^{-\delta t}$ which is decreasing exponentially with time over many oscillations, and the oscillating angular frequency is $\sqrt{\omega^{2}-\delta^{2}}$. The galvanometer in such a case issaid to be under-damped $[5,17,18,19]$ or ballistic galvanometer.

\section{Conclusion}

In this paper, an attempt has been made to exemplify the Rohit transform for discussing the theory of a permanent magnet moving coil (PMMC) instrument. This paper brought up the Rohit transform as a powerful mathematical tool for determining the response of a PMMC instrument. The response obtained is the same as obtained with other the methods or approaches [1-5, 14].

\section{References}

[1] Basic Electrical Engineering, C. L. Wadhwa. Publisher: New Age International Pvt. Ltd. $2^{\text {nd }}$ edition, 2011.

[2] Electrical Measurements and Measuring Instruments by U. A. Bakshi and A.V. Bakshi. Publisher: TechnicalPublications, 2008.

[3] A Text Book of Engineering Physics by M.N. Avadhanulu and P.G. Kshirsagar.Publisher: S. Chand Publishing, $11^{\text {th }}$ edition, 2018.

[4] The Physics of Wave and Oscillations by N.K. Bajaj. Publisher: Tata Mc-Graw Hill Publishing Co. Ltd., 1988.

[5] Rohit Gupta, Rahul Gupta, Residue approach to mathemat ical analy sis of the moving coil galvanometer, International Journal of Advanced Trends in Engineering and Technology, 4(1), 2019, pp. 06-10.

[6] Rohit Gupta, On Novel Integral Transform: Rohit Transform and Its Application to Boundary Value Problems, "ASIO Journal of Chemistry, Physics, Mathematics and Applied Sciences", 4(1), 2020: 08-13.

[7] Rohit Gupta, Rahul Gupta, Dinesh Verma, Solving Schrodinger equation for a quantum mechanical particle by a new integral transform: Rohit Transform, "ASIO Journal of Chemistry, Physics, Mathematics and Applied Sciences", 4(1), 2020: 32-36.

[8] Rohit Gupta, Rahul Gupta, Analysis of RLC circuits with exponential excitation sources by a new integral transform: Rohit Transform, "ASIO Journal of Engineering and Technological Perspective Research", 5(1), 2020,pp.22-24.

[9] Rohit Gupta, Yuvraj Singh, Dinesh Verma, Response of a basic series inverter by the application of convolution theorem, "ASIO Journal of Engi. and Technological Perspective Research", 5(1), 2020,pp. 14-17.

[10] Anamika, Rohit Gupta, Analysis Of Basic Series Inverter Via The Application Of Rohit Transform, "International Journal of Advance Research and Innovative Ideas in Education”,6(6), 2020, pp. 868-873.

[11] Loveneesh Talwar, Rohit Gupta, Analysis of Electric Net work Circuits with Sinusoidal Potential Sources via Rohit Transform, International Journal of Advanced Research in Electrical, Electronics and Instrument at ion Engineering, Volume 9, Issue 11, November 2020, pp. 3929-3023.

[12] Neeraj Pandita, Rohit Gupta, Analysis Of Uniform Infinite Fin Via Means Of Rohit Transform, "International Journal Of Advance Research And Innovative Ideas In Education", 6(6), 2020, pp. 1033-36.

[13] Neeraj Pandita and Rohit Gupta,Heat Conducted Through Fins Of Varying Cross-Sections Via Rohit Transform, "EPRA Journal Of Research And Development”, 5(12), 2020, pp. 222-226.

[14] A Study of Electromagnet Moving Coil Galvanometers for Use in Alternating-current Measurements by Ernest Edward Weibel. Publisher: U.S. Government Printing Office, 1918.

[15] Rahul Gupta and Rohit Gupta, Impulsive Responses of Damped Mechanical and Electrical Oscillators, "International Journal ofScientific and Technical Advancements”, 6(3), 2020, pp. 41-44.

[16] Rahul Gupta, Rohit Gupta, Dinesh Verma, Application of Convolution Method to the Impulsive Response of A Lightly Damped Harmonic Oscillator, International Journal of Scientific Research in Physics and Applied Sciences, Vol.7, Issue.3, pp.173-175, June (2019).

[17] Rohit Gupta, Rahul Gupta, Sonica Rajput, Analysis of Damped Harmonic Oscillator by Matrix Method, Inter. Journal of Research and Analytical Reviews, Volume 5, Issue 4, October 2018,pp.479-484.

[18] Rahul Gupta, Rohit Gupta, Dinesh Verma, Application of Novel Integral Transform: Gupta Transform to Mechanical and Electrical Oscillators, "ASIO Journal of Chemistry, Physics, Mathematics and Applied Sciences", 4(1), 2020: 04-07.

[19] Engineering Physics by R.K. Gaur and S.L. Gupta. Publisher: Dhanpat Rai publications, $8^{\text {th }}$ edition, 2008. 